\title{
A Method of Calculating Waist Width of Angle Steel Tower Based On VC++ and OSG
}

\author{
Changzhi Wang \\ China Electric Power Research Institute \\ Beijing, China \\ E-mail: wangchangzhi@epri.sgcc.com.cn \\ Junke Han \\ China Electric Power Research Institute \\ Beijing, China \\ E-mail: hjk@epri.sgcc.com.cn \\ Qinghua Li \\ China Electric Power Research Institute \\ Beijing, China \\ E-mail: lqh@epri.sgcc.com.cn
}

\author{
Fei Wang \\ China Electric Power Research Institute \\ Beijing, China \\ E-mail: wangfei@epri.sgcc.com.cn \\ Xiao Tian \\ China Electric Power Research Institute \\ Beijing, China \\ E-mail: tianxiao@epri.sgcc.com.cn
}

\begin{abstract}
The power transmission tower is a key part of the transmission line, the waist width shall be calculated correctly during two dimensional drawing and tower lofting, which is the fundamental to correct fabrication and assembly of the steel tower. In this paper, we deeply analyzed the connection mode of the principal in the variable slope tower body and the tower section of different size, on the basis of deep analysis of the alignment principle of the rod member main materials in the angle steel tower, calculation of the waist width at every tower section is solved through the double tangent circle method. In this way, we calculated the waist widths of the variable slope multiple tower bodies and the unchanged slope multiple tower sections accurately when ridges aligned. The method is tested and verified in several projects and the corresponding calculation program is written by $\mathrm{VC}++$. Experiments in several projects show that the method is stable and reliable, which can meet the demand of the engineering well.
\end{abstract}

Keywords-Angle steel tower, Calculation of waist width, Double tangent circles, $\mathrm{VC}++$

\section{INTRODUCTION}

By the end of 2015, the length of UHV transmission lines which are in operation or under construction exceeded 22,000 kilometers, the capacity of power transformation (current conversion) exceeds to more than 2.3 million $\mathrm{KV} \cdot \mathrm{A}$, and the transmission capacity of the provincialcross and region-cross has reached to 573 million $\mathrm{kW} \cdot \mathrm{h}$, Based on the data above, China has been initially built into a special high-voltage backbone network and strong smart grid. As a key part of $\mathrm{HV}$ and UHV, the transmission tower lays a solid foundation to the safety and stability of the grid. Due to mature technology, convenient transportation and construction, and cheap material [1], the angle steel tower is widely used in $\mathrm{HV}$ and $\mathrm{UHV}$, so the design accuracy of angle steel tower not only directly affects the postprocessing and installation at site, but also is related to the safety and stability of the transmission line.

The production and fabrication of the power transmission tower consists of the structure calculation and analysis, drawing of the two-dimensional graph, drawing of the assembly graph, tower lofting, drawing of parts, production and trial assembly etc[2]. Every phase, from structure calculation and analysis to tower lofting of the steel tower, is related to the waist width of the steel tower, which has a significant meaning to calculating the size of the steel tower accurately. In this paper, we develop a general method of calculating waist width by computer graphic algorithm based on deep study and analysis of the calculation principle of the waist width of the main steel in the angle steel tower, and the corresponding program is also compiled, which generates good economic benefit during actual production.

\section{CALCUlation PRINCIPLE}

\section{A. General}

In the common angle steel tower, the double main materials and the four main materials directly take the geometrical centre of several rod members as the alignment lines, there is no complicate calculation exists during calculation of the waist width, and the waist width can be determined according to principle of the single line chart. One difficult during calculation of the waist width of the single angle steel main material is alignment of the external surface, the target is to calculate the reasonable waist width of the reference line and ensure external surfaces of the upper align with the lower tower sections aligned[3] 
(facilitate to connect the upper and lower main materials by the clad steel sheet and inner lined angle steel).

The angle steel consists of the several tower bodies, every tower body is divided into several tower sections according to different specification of the main material, calculation of the waist width is generally carried out following sequence from upper area to lower area, the upper and lower waist widths of every tower body and the tower section are calculated one by one, its calculation process is shown as reference figure 1.

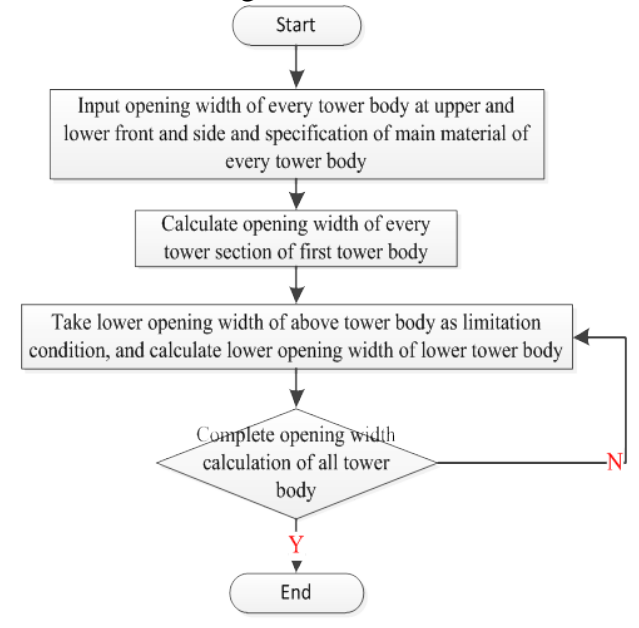

Figure 1 Calculation process of waist width

When it is observed from front and side, relationship between the reference line of the main material angle steel and the outer surface line is shown as figure 2. Red line means the reference line of the angle steel, the black line means the outer surface line of the angle steel. For the large size angle steel with several reference lines, the basic reference line is taken as the alignment line.

Every tower body may include several tower sections; the adjacent tower body generally includes the variable slope. And there is no variable slope between every tower section in the tower body. Before the waist width is calculated, the upper and lower waist widths at utmost top tower body, the lower waist width of every lower tower body, type of the main material and height of the section of every tower section shall be determined. The upper and lower waist widths of every tower body shall be calculated in first step, the upper and lower waist widths of every tower section in the tower body are calculated in second step.
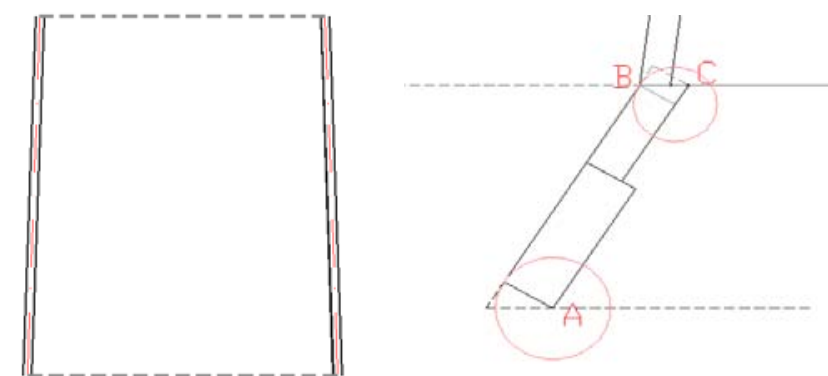

Figure 2 Relationship between reference line of angle steel and outer surface line
Figure 3 Reference calculation of waist of tower body

\section{B. Reference calculation of waist width of tower body}

Input conditions for calculation of the waist width are the upper and lower waist widths of every tower body, it must ensure the lower waist width of the outer surface at the upper tower body at connection location of the adjacent tower body and the upper waist width of the outer surface of the lower tower body is same. But input waist width is waist width of the reference line, it means the following calculation principles must be met at connection location of the tower bodies at the upper and lower sections so as to reach alignment effect of the outer surfaces:

- The upper waist isn't changed, and the lower waist is changed: width of the lower waist of the tower body at upper section is taken as input condition, with of the upper waist of the tower body at lower section is determined in reference to width of the lower waist of the tower body at upper section;

- The lower waist isn't changed, and the upper waist is changed: width of the upper waist of the tower body at lower section is taken as input condition, with of the lower waist of the tower body at upper section is determined in reference to width of the upper waist of the tower body at lower section;

Calculation of the waist width generally applies the principle with the unchanged upper waist and the changed lower waist, calculation principle under such conditions is shown as figure 3 . Considering from the computer graphics, point $\mathrm{A}$ and point $\mathrm{B}$ are known conditions, and point $\mathrm{C}$ is the calculation result.

The point $A$ is actually the reference waist width point of the tower body at the lower section, the point $B$ is the outer surface waist width point of the tower body at the upper section, it can be calculated through the upper and lower reference waist width points of the tower body at the upper section, calculation method is the double external tangent circle method. Its essence is to take the reference line waist width point at the upper and lower waists of some tower bodies as the circle centre, two circles are established taking reference distance of the main materials at the upper and lower sections of the tower body as the radiuses, external common tangent lines of two circles are the outer surface lines of this tower body.

\section{Calculation of waist width of tower section}

For every tower section in the tower body, input conditions for calculation are A and B positions, height of every tower section and the main material, target is to determine position of the node of the main material of every tower section, shown as figure 4 . If the tower body includes two tower sections, the point $\mathrm{C}$ and $\mathrm{D}$ on the connection plane of two tower sections shall be calculated, in which $\mathrm{C}$ is the waist width point of the lower reference line at the upper tower section, $\mathrm{D}$ is the waist width point of the upper reference line at the lower tower section, these two points have the same elevation.

Calculation methods of the $\mathrm{C}$ and $\mathrm{D}$ points also apply the double tangent circle method. The angle between the 
outer surface line and its horizontal plane is determined according to two external tangent circles at point $\mathrm{B}$ and point $\mathrm{A}$, the straight lines $\mathrm{BC}$ and $\mathrm{DA}$ are actually parallel to the outer surface line, and positions of $C$ and $D$ points can be calculated according to Pythagorean theorem.

If there are more tower sections, the node of every tower section shall be calculated, and the reference line waist width point of every tower section shall be calculated for several times according to the above methods.

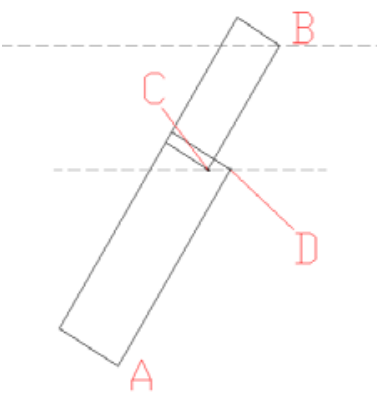

Figure 4 Calculation figure of waist width of tower section width of tower body

\section{DESIGN AND ANALYSIS OF ALGORITHM}

In theory, in order to realize alignment of the outer surface of every tower body at crossing location, the upper waist width of the tower body at the lower section is reckoned according to the lower waist width of the tower body at upper section, the lower waist width of the tower body at the upper section is reckoned according to the upper waist width of the tower body at lower section. This paper takes the most common basis of the lower waist width of the tower body at the upper section reckoning the upper waist width of the tower body at lower section as sample to study and analyze the waist width calculation of the tower body and the tower section.

A. Abbreviations and Acronyms Calculation of upper waist width of tower body at lower section according to lower waist width of tower body at upper section

Graphics shown in figure 5 is drawn according to assumption with variable slope at connection location of the tower body and no variable slope between the tower sections in the tower body specified in this paper. From the graphics, SW (waist width at upper) and XZ (waist width of reference line at lower section) are known conditions, the target is to calculate SZ (waist width of reference line at upper) and XW (waist width at lower).

Because the standard distance of every tower section is known, positions of SZ and XW can be calculated if 1, $\angle 2$ and $\angle 3$ in figure 5 can be calculated, the calculation method is shown as following:

(1) $\angle 2$ can be calculated according to formula $\operatorname{Cos} \angle 2=X Z \mathrm{~J} /$ $\operatorname{Dist}(S W, X Z)$;

(2) $\angle 1$ is calculated by Cosine function according to positions of $X Z$ and $S W$;
(3) $\angle 3=P I-\angle 1-\angle 2, \angle 3$ is calculated;

(4) $X W$ is calculated according to $\operatorname{Dist}(X Z, X W)=X Z J / \operatorname{Cos} \angle 3$,

(5) $S Z$ is calculated according to Dist $(S Z, S W)=S Z J / \operatorname{Cos} \angle 3$.

B. Node calculation of every tower section in tower body

Graphics shown in figure 6 is drawn according to assumption with variable slope at connection location of the tower body and no variable slope between the tower sections in the tower body specified in this paper. Seen from the graphics, SZ (waist width of reference line at upper) and $\mathrm{XZ}$ (waist width of reference line at lower) are known conditions, the target is to calculate SW (external waist width at upper) and XW (external waist width at lower) and the middle nodes $\mathrm{B}$ and $\mathrm{C}$ of the tower section.

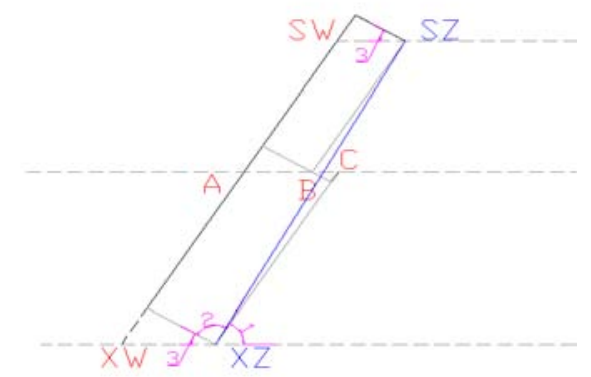

Figure 6 Calculating node of every tower section

Because the standard distance of every tower section is known, positions of SW and XW can be calculated if 1, 2 and 3 angles in figure 6 can be calculated, calculation method is shown as following:

(1) $\angle 2$ can be calculated according to formula $\operatorname{Cos} \angle 2=(X Z J$ - SZJ) / Dist(SZ, XZ);

(2) $\angle 1$ is calculated by Cosine function according to positions of $X Z$ and $S W$;

(3) $\angle 3=P I-\angle 1-\angle 2, \angle 3$ is calculated;

(4) $X W$ is calculated according to $\operatorname{Dist}(X Z, X W)=X Z J / \operatorname{Cos} \angle 3$,

(5) $S W$ is calculated according to Dist(SZ, SW) $=S Z J / \operatorname{Cos} \angle 3$.

(6) Point A1 is calculated as the linear interpolation according to relationship between heights of the tower section;

(7) Point $B$ is calculated according to $\operatorname{Dist}(A, B)=S Z J /$ $\operatorname{Cos} \angle 3$.

(8) Point $C$ is calculated according to $\operatorname{Dist}(A, C)=X Z J /$ $\operatorname{Cos} \angle 3$.

\section{Calculation of waist width at front and side}

Because characteristics of the single main material angle steel tower are known, when it is observed from front face, one limb of the angle steel is vertical to observation direction, another limb is parallel to observation direction (it means there is waist and closing angle on the main material). There are same conditions when the tower body is observed from side, therefore the two dimensional calculation method described in this paper can meet calculation requirements of the model, the algorithm described in this paper is 
implemented once during calculation of the waist width at front and side.

\section{CONCLUSION}

According to the algorithm in this paper, the author writes the corresponding calculation program by $\mathrm{VC}++$ and OSG [4] (OpenSceneGraph) three dimensional visit engine during research of this project, this program includes input and output windows for calculation of the waist width, and OSG is applied to develop the three dimensional visualization display environment. Calculation quantity of the algorithm described in this paper is very small, all waist width calculations of the complicate tower type are complete within 1 second, figure 7 is the single line figure obtained from calculation of the waist width ( display platform is MFC+OSG[5]).

Figure 8 is the split joint effect at connection location of two tower bodies. Seen from the figure, the algorithm in this paper can ensure that alignment of the external surface of the main material steel angle is realized well at the variable slope location of the tower body

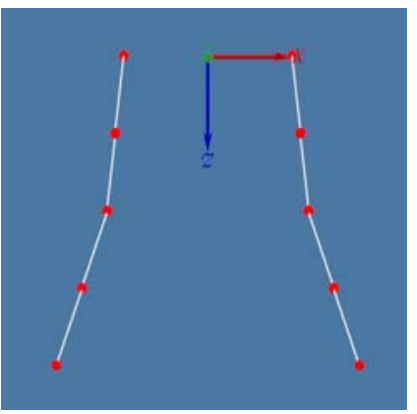

Figure 7 Result of waist width calculation single line.

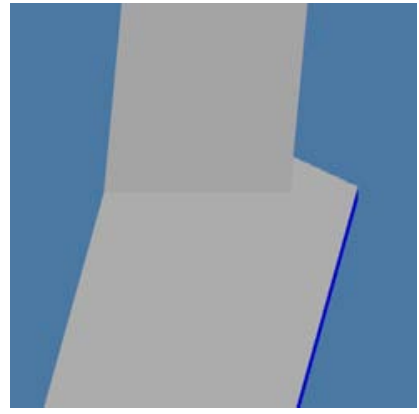

Figure 8 Split joint of main material figure
Taking the waist width calculation of the angle steel tower as basis, the author writes the software program which is applicable to modelling of the angle steel tower. It can quickly display the structure of the whole tower, and various fabrication drawings can be output according to demand, figure 9 is a local model of the steel tower which is modelled by this software. At present, the result of the waist width has been applied in several tower design projects, which is accurate and reliable. And the calculation method is simple and easy to understand, which has a good extension perspective.

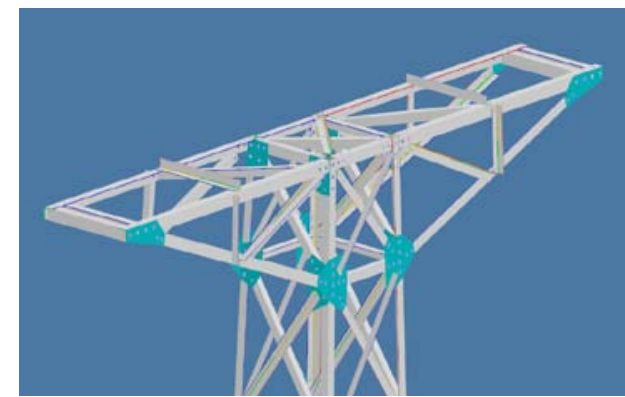

Figure 9 Local model of steel tower

\section{References}

[1] Application and Dissemination of Steel Tubular Tower in Transmission Lines[J] , SunZhusen,ChengYongfeng,ZhangQiang, Power System Technology,2010.6;

[2] Application of 3D CAD Lofting Technique in The Iron Manufacturing Enterprise[J], YiXuezhi, Equipment Manufacturing Technology,2006.04;

[3] Automatic Assembly in 3D Lofting of Iron Tower,LiZexue,WuQingwen[J],LiMing, Journal of Computer-Aided Design \& Computer Graphics,2006.07.

[4] Open Scene Graph 3D rendering engine programming guide[M], XiaoPeng,LiuGengdai, XuMingliang, Tsinghua University Press,2010.01;

[5] Open Scene Graph 3D rendering engine design and practice[M],WangRui,QianXuelei , Tsinghua University Press , 2009.11; 